\title{
Quality modeling of drinking groundwater using GIS in rural communities, northwest of Iran
}

\author{
Mohammad Mosaferi ${ }^{1}$, Mojtaba Pourakbar ${ }^{2 *}$, Mohammad Shakerkhatibi ${ }^{3}$, Esmaeil Fatehifar ${ }^{4}$ and Mehdi Belvasi ${ }^{5}$
}

\begin{abstract}
Given the importance of groundwater resources in water supply, this work aimed to study quality of drinking groundwater in rural areas in Tabriz county, northwest of Iran. Thirty two groundwater samples from different areas were collected and analyzed in terms of general parameters along with 20 heavy metals (e.g. As, Hg and ...). The data of the analyses were applied as an attribute database for preparing thematic maps and showing water quality parameters. Multivariate statistical techniques, including principal component analysis (PCA) and hierarchical cluster analysis (CA) were used to compare and evaluate water quality. The findings showed that hydrochemical faces of the groundwater were of calcium-bicarbonate type. EC values were from 110 to $1750 \mu \mathrm{s} / \mathrm{cm}$, in which concentration of salts was high in the east and a zone in north of the studied area. Hardness was from 52 to $476 \mathrm{mg} / \mathrm{l}$ and $\mathrm{CaCO}_{3}$ with average value of $185.88 \pm 106.56 \mathrm{mg} / \mathrm{L}$ indicated hard water. Dominant cations and anions were $\mathrm{Ca}^{2+}>\mathrm{Na}^{+}>\mathrm{Mg}^{2+}>$ $\mathrm{K}^{+}$and $\mathrm{HCO}_{3}{ }^{-}>\mathrm{Cl}^{-}>\mathrm{SO}_{4}{ }^{2-}>\mathrm{NO}_{3}{ }^{2}$, respectively. In the western areas, arsenic contamination was observed as high as $69 \mathrm{\mu g} / \mathrm{L}$. Moreover, mercury was above the standard level in one of the villages. Eskandar and Olakandi villages had the lowest quality of drinking water. In terms of CA, sampling sites were classified into four clusters of similar water quality and PCA demonstrated that 3 components could cover $84.3 \%$ of the parameters. For investigating arsenic anomaly, conducting a comprehensive study in the western part of studied area is strongly recommended.
\end{abstract}

Keywords: Heavy metals, GIS, Groundwater, Mapping, Multivariate statistic

\section{Background}

Safe drinking water is one of the necessities in sustaining life and a satisfactory (adequate, safe and accessible) supply of water must be available for all people. The advantage of groundwater is that it can be abstracted in many places, which makes pipe transportation unnecessary. Furthermore, water is hygienically reliable and generally it has a constant composition. Sometimes, it can be even distributed without any treatment although a simple and cheap treatment (e.g. disinfection) is often inevitable [1-4].

Due to the rising demand for clean drinking water, management of groundwater quality, especially in developing countries, is very important. It has been reported that approximately one third of the world's population use groundwater for drinking. Thus, sophisticated monitoring of quality of such resources would play a key role

\footnotetext{
* Correspondence: ppourakbar@yahoo.com

${ }^{2}$ School of Health, Student research committee, Tabriz University of medical sciences, Tabriz, Iran

Full list of author information is available at the end of the article
}

in achieving globally sustainable development in near future [5-7].

After assessing groundwater, it is important to simplify results of the study for policy makers and other stakeholders. So, nowadays, using other sciences such as geographical information system (GIS) has been increased. GIS is a management tool that has grown since the late 20th century. In the past 10 years, the number of GIS users has substantially increased. GIS technology has previously facilitated laborious procedures [8-10]. During the past two decades, various researchers have reported its application in groundwater modeling and quality assessment. Balakrishnan et al. demonstrated spatial variations in groundwater quality using GIS and groundwater quality information maps of the entire studied area in India [11]. Jamshidzadeh and Mirbagheri examined quality and quantity changes in an aquifer in central Iran. Accordingly, using 53 observation wells showed that mean water table declined $0.496 \mathrm{~m} /$ year. Also, most of the water samples which were used for quality analysis were not potable [12]. Contamination of groundwater in an area of $180 \mathrm{~km}^{2}$ in 
India was studied by Arumugam and Elangovan; then, they analyzed major anions and cations and found that most of the locations were contaminated by high levels of $\mathrm{EC}$, TDS, $\mathrm{K}$ and $\mathrm{NO}_{3}$ [4]. Another study which was performed by ThiHanh Hoang et al. during 2007-2008 in Mekong River delta in Vietnam found that 26\%, 74\% and $50 \%$ of groundwater samples were above the drinking water guidelines of United State Environmental Protection Agency (USEPA) as far as As (10 mg/L), Mn (0.05 mg/L) and $\mathrm{Fe}(0.3 \mathrm{mg} / \mathrm{L})$ were concerned, respectively [13].

In the present work, drinking groundwater quality was surveyed and modeled in rural communities of Tabriz in northwest of Iran using GIS. The villages located in Tabriz were of great importance since they had high population compared with other counties in East Azerbaijan province. Uncontrolled expansion of industries, agriculture, settlement and deficiencies in waste management and disposal are considered the threat to surface and groundwater [14]. Moreover, juxtaposition of rural communities with industrial areas around Tabriz county is by itself a threat for the quality of drinking water. There were about 70 villages in Tabriz, only 46 of which were under the coverage of East Azerbaijan Rural Water and Wastewater Company (EARWWC). In these villages, drinking water was mainly provided from groundwater (spring, well or qanat) and water distribution system of Tabriz county was used in some parts. The objective of present study was to evaluate drinking groundwater quality using different method such as mapping of quality parameters along with heavy metals of concern, multivariate statistical analysis to classify water resources and determining hydrochemical faces of the groundwater.

\section{Methods}

\section{Studied area}

Tabriz is located in central part of East Azerbaijan province with the area of about $2167.2 \mathrm{~km}^{2}$ which is $4.76 \%$ of total area of the province (Figure 1). It is subdivided into two districts: central district and Khosrowshahr district with five cities: Tabriz, Basmenj, Sardrud, MalekKian and Khosrowshahr. Tabriz, as the center of this province, is the fifth largest city and one of the historical capitals of Iran which is situated at the altitude of $1,350 \mathrm{~m}$ above sea level.

\section{Water sampling and analysis}

Drinking water of the villages around Tabriz was provided from either groundwater sources (wells, springs and qanats) or urban distribution system. Only the villages using groundwater sources which were under the coverage of EARWWC were included in this study.

In order to assess groundwater quality, sampling was done in 2012 and 32 samples were totally gathered during the study. The samples were collected in acid-washed PET bottles after $5 \mathrm{~min}$ discharge of the current and 3 times washing of the bottles. Parameters including $\mathrm{pH}$, electric conductivity (EC), hardness, major cations $\left(\mathrm{Ca}^{2+}\right.$, $\mathrm{Mg}^{2+}, \mathrm{Na}^{+}$and $\left.\mathrm{K}^{+}\right)$and major anions $\left(\mathrm{HCO}_{3}{ }^{-}, \mathrm{SO}_{4}{ }^{2-}\right.$,

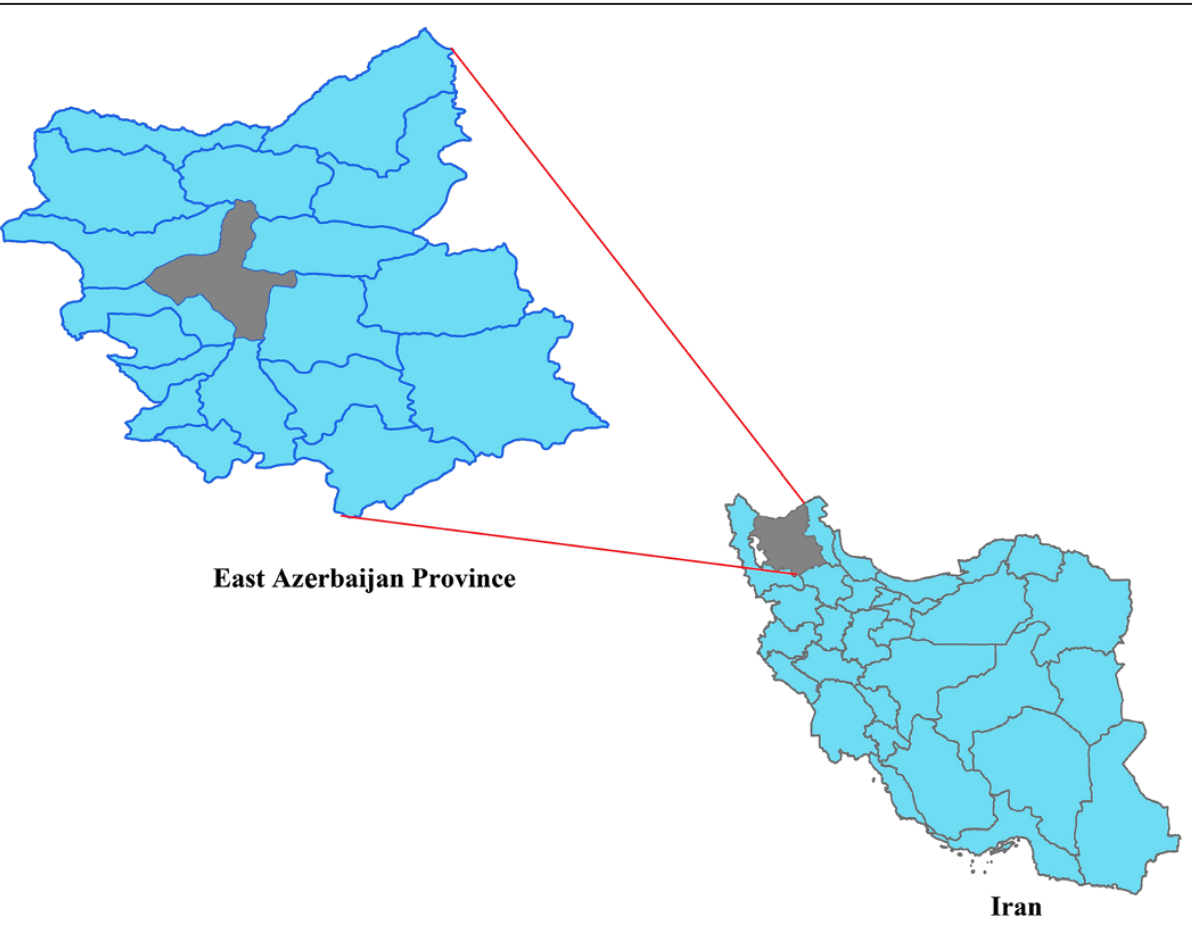

Figure 1 Location of the studied area (Tabriz) on Iran map. 
$\mathrm{Cl}^{-}, \mathrm{F}^{-}$and $\mathrm{NO}_{3}{ }^{2-}$ ) were considered to be measured. All the analyses were according to standard methods for examination of water and wastewater [15]. within addition to the above mentioned parameters, heavy metals such as arsenic, aluminum, boron, barium, beryllium, chromium, copper, mercury, tin, zink, cadmium, cobalt, iron, manganese, molybdenum, nickel, lead, antimony, selenium and vanadium were analyzed by ICPOES (inductively coupled plasma optical emission spectrometry) method. Due to some financial limitations, only 18 out of 32 samples were analyzed in terms of the presence of heavy metals. In order to applicability of the research, samples for heavy metal analysis were selected according to the recommendations made by experts of the EARWWC considering previous available analysis results made by EARWWC. Also, population of village was considered as another important criteria for selected villages.

All the data were entered into a spatial database and spatial variations of the results were developed using inverse distance weighting (IDW) method. Arc GIS software (version 10.0) was also applied for developing maps. IDW interpolation assumes that each measured point has a local influence that diminishes with distance. Thus, points in the near neighborhood are given high weights, whereas points at a far distance are given small weights. The general formula of IDW interpolation for 2-D problems is the following:

$$
w(x, y)=\sum_{i=1}^{N} i_{i} w_{i} \quad{ }_{i}=\frac{\left(\frac{1}{d_{i}}\right)^{p}}{\sum_{i=1}^{N}\left(\frac{1}{d_{i}}\right)^{p}}
$$

Where $w(x, y)$ is the predicted value at location $(x, y), N$ is the number of nearest known points surrounding $(x, y), \lambda_{i}$ are the weights assigned to each known point value $\mathrm{w}_{\mathrm{i}}$ at location $\left(x_{i} y_{i}\right), d_{i}$ are the 2-D Euclidean distances between each $\left(x_{i} y_{i}\right)$ and $(x, y)$, and $\mathrm{p}$ is the experiment which influences the weighting of $w_{i}$ on $w$ [16]. The advantage of IDW is that it is intuitive and efficient, that's why IDW method is widely used in spatial interpolation of groundwater quality [11].

In order to clarify results of this work, piper diagrams were plotted using AqQa software and then interpreted.

\section{Multivariate statistical analysis}

Descriptive statistics (including means, maximum, minimum and standard deviations), logistic regression model, correlation and analysis of variance (ANOVA) tests were done to analyze water quality data and their relationships. Also, principal component analysis (PCA) method (rotation method Varimax, Kaiser Normalization) and cluster analysis (CA) were used to group the related water quality parameters. Varimax rotation is the most widely used orthogonal rotation in PCA for easier interpretation of results [17-19]. Hierarchical agglomerative clustering is the most common approach which provides instinctive similarity relationships between any one sample and the entire dataset and is typically illustrated by a dendrogram (tree diagram) [20].

\section{Results and discussion}

\section{Physicochemical analysis}

Results of the hydrochemical analysis along with descriptive statistics of quality parameters of the groundwater samples which were taken from the studied area are presented in Table 1.

EC values in the investigated area ranged from 110 to $1750 \mu \mathrm{s} / \mathrm{cm}$, these values are much less than the values reported by Baghvand et al. in an aquifer in Iran central desert (1987-12751 $\mu \mathrm{s} / \mathrm{cm})$ [7]. Figure 2 represents EC variation in Tabriz, indicating high concentration of salts in east and a zone in north of the studied area. In terms of hardness, water is grouped as soft water $(<75 \mathrm{mg} / \mathrm{L}$ $\left.\mathrm{CaCO}_{3}\right)$, medium hard (75-150 mg/L $\left.\mathrm{CaCO}_{3}\right)$, hard water (150-300 mg/L $\mathrm{CaCO}_{3}$ ) and very hard water $(>300 \mathrm{mg} / \mathrm{L}$ $\mathrm{CaCO}_{3}$ ). Range and average values of total hardness in the sampled water were 52 to 476 and $185.88 \pm 106.56 \mathrm{mg} / \mathrm{L}$ as $\mathrm{CaCO}_{3}$, respectively, representing that the studied water samples could be grouped as hard water (Figure 3). In a similar study in India, majority of the samples fall in very hard water category $\left(>300 \mathrm{mg} / \mathrm{L} \mathrm{CaCO}_{3}\right)$ [11]. Nitrate values, as an important parameter regarding its health effects, varied from 0.4 to $59.4 \mathrm{mg} / \mathrm{L}$ with average value of $11.91 \pm 10.49$. In the similar study in India, Nitrate concentration in $73.68 \%$ of samples exceeded the guideline value (50 mg/L) [11]. Nitrate distribution is given in Figure 4, according to which only, in village 2 (Eskandar), level of nitrate exceeded guideline value $(50 \mathrm{mg} / \mathrm{L})$. Figures $5,6,7$ show $\mathrm{pH}$, bicarbonate and fluoride variations in the studied area, respectively. As can be seen, in most parts of the studied area, $\mathrm{pH}$ values were above 7; however, in some parts like north, east and southwest, this value was below 7 . In many parts of the considered area, bicarbonate concentration was below $250 \mathrm{mg} / \mathrm{L}$ as $\mathrm{CaCO}_{3}$. For fluoride, concentration was less than $0.5 \mathrm{mg} / \mathrm{L}$, except in villages 3 and 28 .

\section{Hydrochemical faces}

Tables 2 and 3 demonstrate percentage of constituents at the sampled points and hydrochemical faces of the groundwater according to piper diagram, respectively. According to these tables, in most parts of the considered area, hydrochemical faces of the groundwater were of calciumbicarbonate type. Piper diagram of the studied area is given in Figure 8. According to the diagram, groundwater was divided to six faces $[4,12,21]$. In the present investigation, bicarbonate was the most dominant anion (in 93\% of the 
Table 1 Hydrochemical analysis of the groundwater samples taken from the study area

\begin{tabular}{|c|c|c|c|c|c|c|c|c|c|c|c|c|c|c|}
\hline \multirow[t]{2}{*}{ No } & \multirow[t]{2}{*}{ Villages } & \multicolumn{4}{|c|}{ Cations (mg/L) } & \multicolumn{5}{|c|}{ Anions (mg/L) } & \multirow[t]{2}{*}{$\mathrm{pH}$} & \multirow{2}{*}{$\begin{array}{c}E C \\
(\mu \mathrm{s} / \mathrm{cm})\end{array}$} & \multirow{2}{*}{$\begin{array}{c}\text { TDS } \\
(\mathrm{mg} / \mathrm{L})\end{array}$} & \multirow{2}{*}{$\begin{array}{r}\text { Hardnes } \\
(\mathrm{mg} / \mathrm{L})\end{array}$} \\
\hline & & $\mathrm{Ca}$ & Mg & $\mathrm{Na}$ & $K$ & $\mathrm{~F}$ & $\mathrm{Cl}$ & $\mathrm{SO}_{4}$ & $\mathrm{HCO}_{3}$ & $\mathrm{NO}_{3}$ & & & & \\
\hline 1 & Hervi & 40.0 & 19.4 & 6.1 & 5.0 & 0.20 & 26 & 16.2 & 175.7 & 0.4 & 6.76 & 367 & 238.6 & 180 \\
\hline 2 & Eskandar & 160.0 & 18.5 & 160.0 & 9.0 & 0.20 & 190 & 164.6 & 414.8 & 59.4 & 6.67 & 1750 & 1137.5 & 476 \\
\hline 3 & Ligvan & 32.0 & 16.5 & 3.6 & 3.0 & 0.60 & 20 & 1.8 & 146.4 & 3.9 & 6.88 & 294 & 191.1 & 148 \\
\hline 4 & Shadabad & 24.0 & 29.2 & 29.5 & 5.0 & 0.20 & 30 & 45.5 & 180.6 & 4.0 & 6.93 & 470 & 305.5 & 180 \\
\hline 5 & Esparakhun & 8.0 & 14.6 & 4.0 & 1.0 & 0.17 & 12 & 2.2 & 78.1 & 2.1 & 7.10 & 165 & 107.3 & 80 \\
\hline 6 & Kondrud & 80.0 & 30.1 & 51.6 & 6.5 & 0.20 & 72 & 74.6 & 297.7 & 9.4 & 6.48 & 837 & 544.1 & 324 \\
\hline 7 & Dizaj leylikhani & 24.0 & 14.6 & 11.0 & 4.0 & 0.15 & 10 & 12.2 & 126.9 & 7.4 & 7.13 & 289 & 187.9 & 120 \\
\hline 8 & Asbes & 40.0 & 29.2 & 86.1 & 5.0 & 0.40 & 94 & 77.6 & 244.0 & 13.5 & 6.81 & 881 & 572.7 & 220 \\
\hline 9 & Asenjan & 88.0 & 19.4 & 71.4 & 6.5 & 0.20 & 124 & 107.6 & 219.6 & 15.0 & 7.20 & 926 & 601.9 & 300 \\
\hline 10 & Varanag & 56.0 & 21.4 & 61.5 & 7.1 & 0.20 & 54 & 60.4 & 258.6 & 10.5 & 6.80 & 664 & 431.6 & 228 \\
\hline 11 & Ola kandi & 44.8 & 70.0 & 87.4 & 16.0 & 0.40 & 30 & 220.5 & 439.2 & 5.4 & 6.89 & 1232 & 800.8 & 400 \\
\hline 12 & Esfahlan & 40.0 & 63.2 & 73.8 & 9.9 & 0.20 & 32 & 195.6 & 302.6 & 15.0 & 6.46 & 1024 & 665.6 & 360 \\
\hline 13 & Anarjan & 40.0 & 34.0 & 22.1 & 4.8 & 0.20 & 36 & 46.4 & 239.1 & 5.2 & 6.09 & 552 & 358.8 & 240 \\
\hline 14 & Komanj olya & 16.0 & 14.6 & 4.5 & 0.9 & 0.20 & 4 & 5.4 & 92.7 & 6.5 & 6.34 & 205 & 133.3 & 100 \\
\hline 15 & Zinjanab & 24.0 & 4.9 & 4.5 & 2.0 & 0.20 & 12 & 9.9 & 53.7 & 5.0 & 7.49 & 168 & 109.2 & 80 \\
\hline 16 & Espiran1 & 40.0 & 26.2 & 137.8 & 9.0 & 0.40 & 110 & 173.9 & 175.7 & 16.2 & 7.41 & 1010 & 656.5 & 208 \\
\hline 17 & Espiran2 & 43.2 & 17.5 & 115.6 & 9.0 & 0.30 & 100 & 179.5 & 146.4 & 11.4 & 7.42 & 930 & 604.5 & 180 \\
\hline 18 & Khellejan & 48.0 & 19.4 & 46.7 & 8.0 & 0.30 & 60 & 49.6 & 209.8 & 9.3 & 6.54 & 685 & 445.3 & 200 \\
\hline 19 & Shadabad olya & 27.1 & 6.6 & 27.7 & 3.4 & 0.22 & 25 & 20.0 & 126.9 & 7.1 & 7.66 & 303 & 197.0 & 96 \\
\hline 20 & Zaranag & 24.0 & 5.7 & 21.0 & 3.2 & 0.23 & 11 & 12.0 & 122.0 & 6.2 & 7.74 & 245 & 159.3 & 84 \\
\hline 21 & Bagh yagub & 41.5 & 15.2 & 41.5 & 4.1 & 0.52 & 25 & 34.0 & 229.4 & 8.4 & 7.55 & 487 & 316.6 & 168 \\
\hline 22 & Nemat abad & 27.1 & 7.6 & 29.6 & 3.5 & 0.23 & 25 & 22.0 & 126.9 & 9.7 & 7.52 & 310 & 201.5 & 100 \\
\hline 23 & Fath abad & 27.1 & 9.5 & 27.9 & 3.4 & 0.18 & 25 & 24.0 & 122.0 & 13.3 & 7.64 & 315 & 204.8 & 108 \\
\hline 24 & Hezar baran & 62.3 & 19.0 & 52.5 & 7.3 & 0.49 & 57 & 55.0 & 258.6 & 12.8 & 7.50 & 667 & 433.6 & 236 \\
\hline 25 & Beirag & 35.1 & 7.6 & 22.8 & 4.4 & 0.34 & 11 & 13.0 & 165.9 & 9.3 & 7.63 & 318 & 206.7 & 120 \\
\hline 26 & Jangur & 22.4 & 7.6 & 24.1 & 3.5 & 0.54 & 14 & 13.0 & 122.0 & 11.1 & 7.71 & 273 & 177.5 & 88 \\
\hline 27 & Chavan & 19.2 & 8.5 & 16.9 & 3.7 & 0.18 & 7 & 8.0 & 117.1 & 13.3 & 8.02 & 230 & 149.5 & 84 \\
\hline 28 & Gollujeh & 12.8 & 4.7 & 3.9 & 0.1 & 0.90 & 4 & 3.0 & 39.0 & 22.1 & 7.12 & 110 & 71.5 & 52 \\
\hline 29 & Nosrat abad & 17.6 & 3.8 & 5.4 & 0.4 & 0.14 & 4 & 4.0 & 58.6 & 17.7 & 7.52 & 131 & 85.2 & 60 \\
\hline 30 & Anakhatun & 44.7 & 15.2 & 22.4 & 3.1 & 0.27 & 32 & 32.0 & 170.8 & 15.9 & 7.76 & 422 & 274.3 & 176 \\
\hline 31 & Karjan & 63.9 & 12.3 & 57.5 & 6.2 & 0.41 & 68 & 70.0 & 190.3 & 28.8 & 7.51 & 678 & 440.7 & 212 \\
\hline \multirow[t]{5}{*}{32} & Aghaj oghli & 61.2 & 44.3 & 91.3 & 4.3 & 0.52 & 107 & 72.3 & 372.5 & 6.1 & 7.64 & 988 & 642.2 & 340 \\
\hline & Maximum & 160.0 & 70.0 & 160.0 & 16.0 & 0.90 & 190 & 220.5 & 439.2 & 59.4 & 8.02 & 1750 & 1137.5 & 476 \\
\hline & Minimum & 8.0 & 3.8 & 3.6 & 0.1 & 0.14 & 4 & 1.8 & 39.0 & 0.4 & 6.09 & 110 & 71.5 & 52 \\
\hline & Mean & 41.7 & 19.7 & 44.4 & 5.1 & 0.31 & 44.6 & 57.1 & 188.2 & 11.9 & 7.19 & 560 & 364.1 & 185.9 \\
\hline & Std. deviation & 28.57 & 15.54 & 40.76 & 3.26 & 0.17 & 43.6 & 63.17 & 99.14 & 10.4 & 0.49 & 382.90 & 248.9 & 106.56 \\
\hline
\end{tabular}

samples) and calcium was the most dominant cation (in $65 \%$ of the samples). Therefore, chemical characteristic of the water was dominated by $\mathrm{Ca} \mathrm{HCO}_{3}$ type water.

\section{Heavy metals}

Table 4 shows concentration of heavy metals and trace elements in the groundwater samples. In addition to the heavy metals presented in Table 4, some other elements including cadmium, cobalt, iron, manganese, molybdenum, nickel, lead, antimony, selenium and vanadium, were either analyzed which their concentrations were non-detectable in samples.

One of the heavy metals of concern in the studied area was arsenic, which is a naturally occurring contaminant 


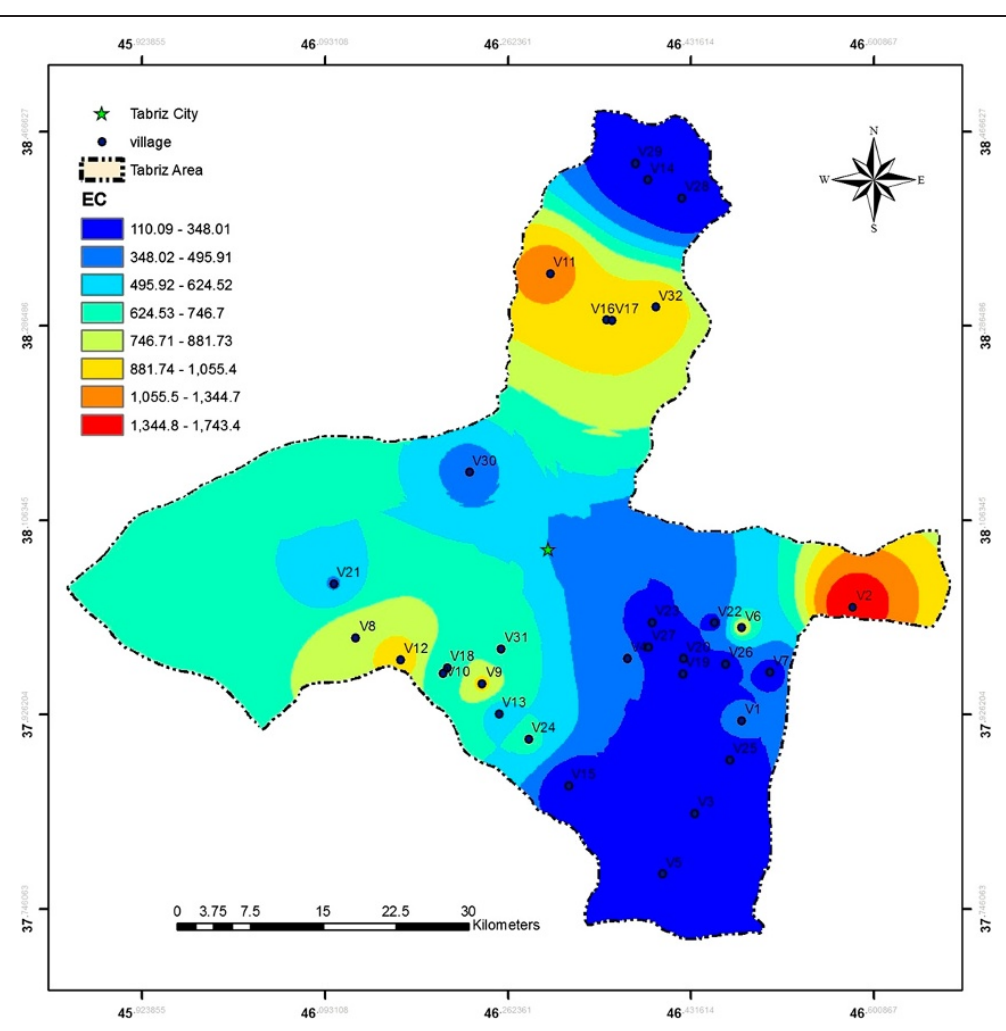

Figure 2 EC variation in the studied area.

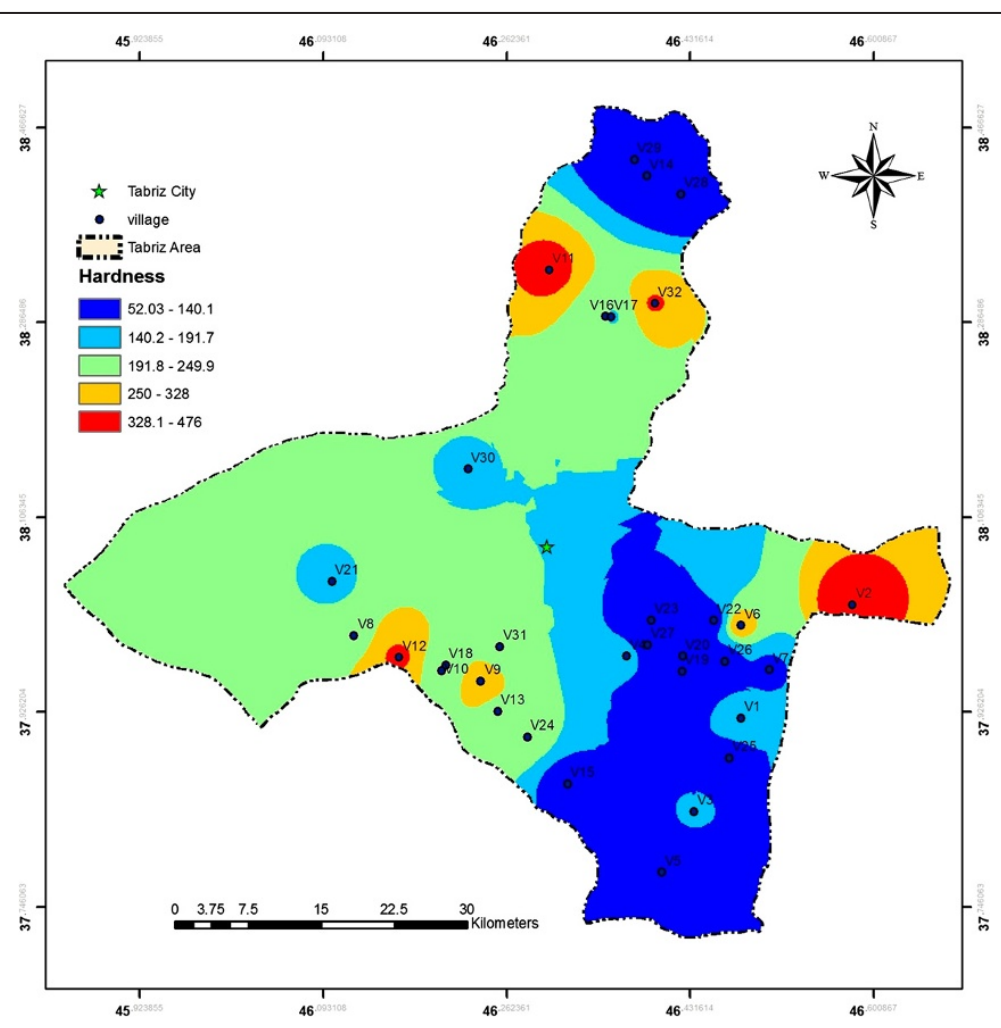

Figure 3 Hardness distribution in the considered area. 


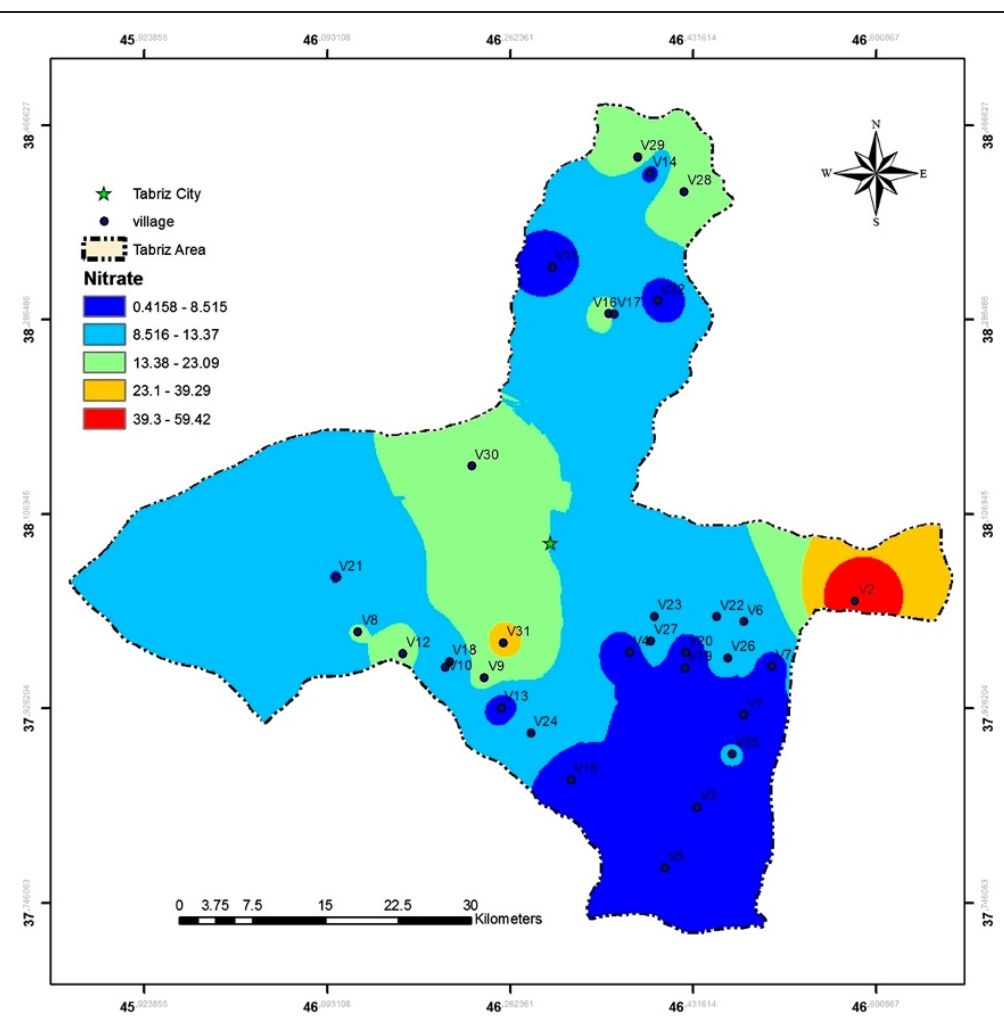

Figure 4 Nitrate distribution in the studied area.

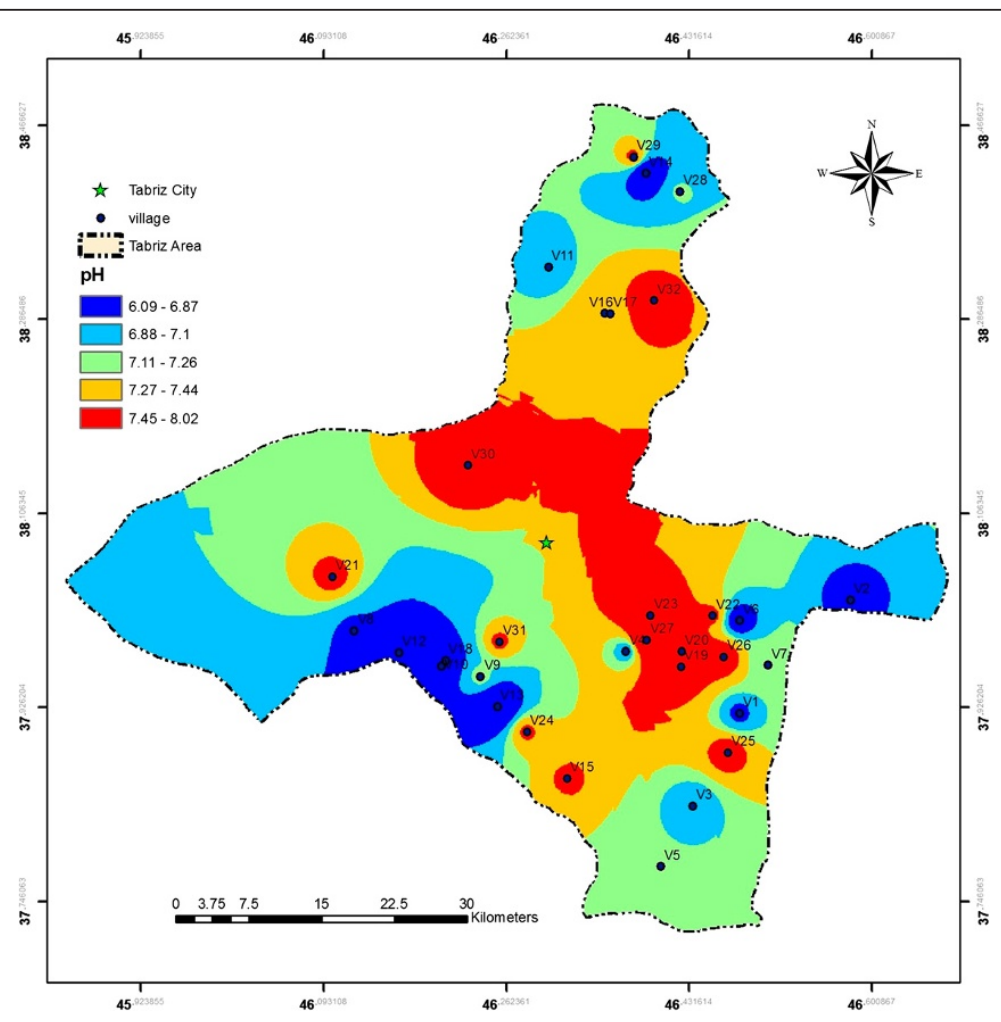

Figure $5 \mathrm{pH}$ distribution in the studied area. 


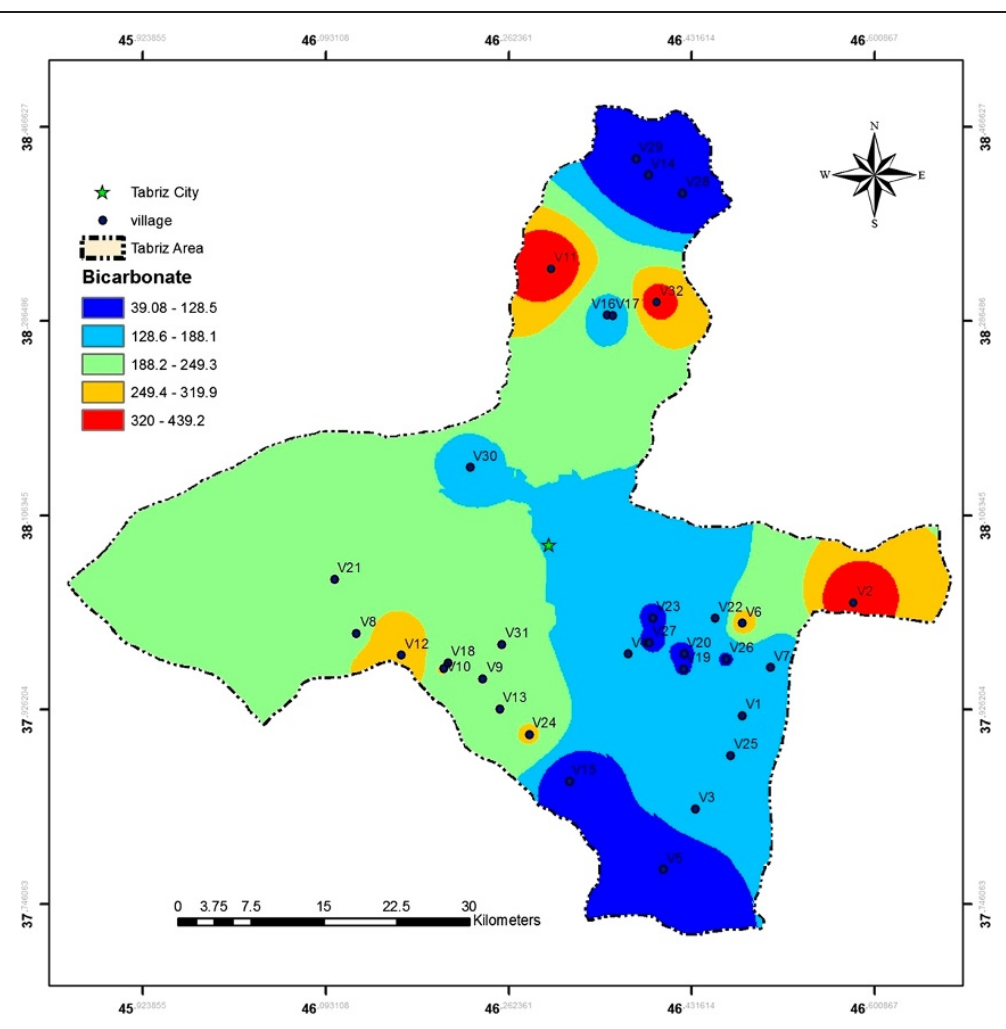

Figure 6 Bicarbonate distribution in the studied area.

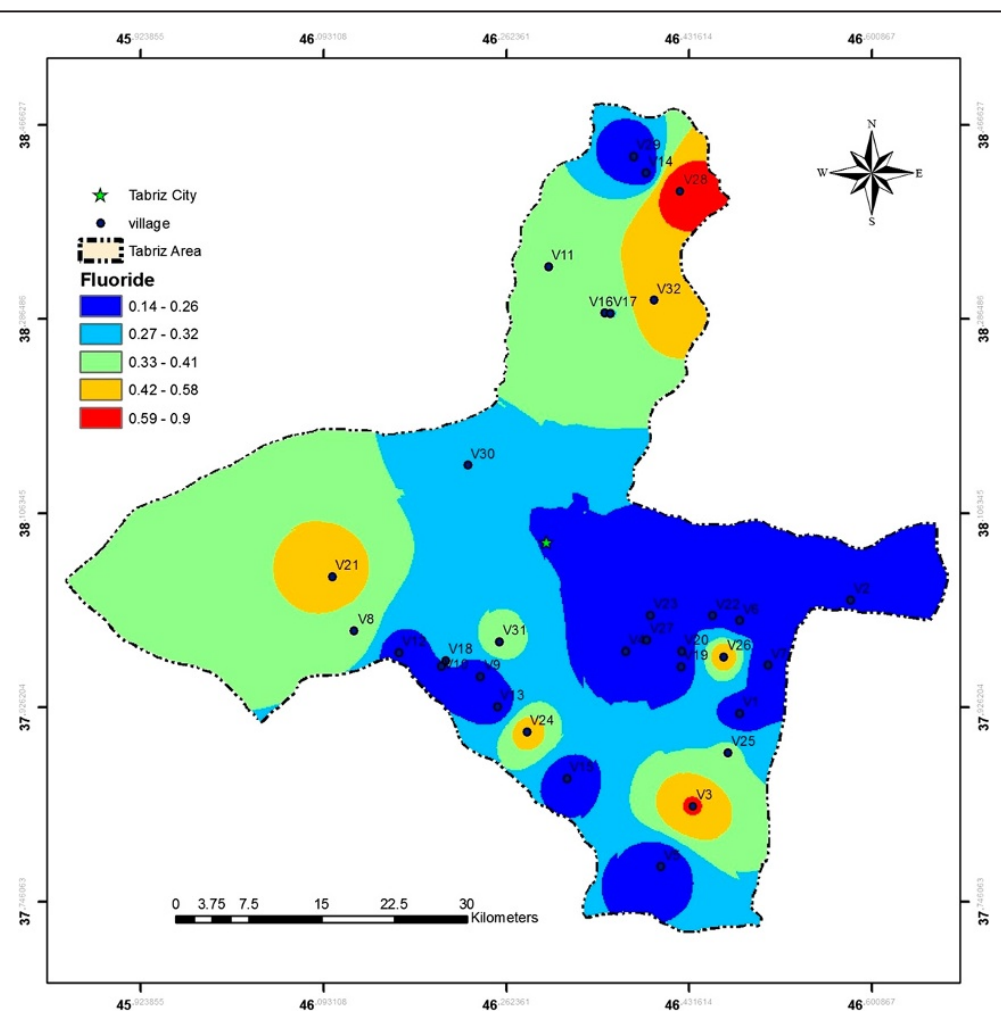

Figure 7 Fluoride distribution in the studied area. 
Table 2 Percentage of constituents of the groundwater samples and ion balance

\begin{tabular}{|c|c|c|c|c|c|c|c|c|c|c|c|c|}
\hline \multirow[t]{2}{*}{ Villages } & \multicolumn{4}{|c|}{ Cations (\%) } & \multicolumn{5}{|c|}{ Anions (\%) } & \multicolumn{3}{|c|}{ Ion balance } \\
\hline & $\mathrm{Ca}$ & $\mathrm{Mg}$ & $\mathrm{Na}$ & $\mathrm{K}$ & $\mathrm{F}$ & $\mathrm{Cl}$ & $\mathrm{SO}_{4}$ & $\mathrm{HCO}_{3}$ & $\mathrm{NO}_{3}$ & $\Sigma$ Cations (meq/l) & $\Sigma$ Anions (meq/l) & Error \\
\hline $\mathrm{V} 1$ & 50.06 & 40.13 & 6.61 & 3.21 & 0.27 & 18.53 & 8.53 & 72.51 & 0.17 & 3.99 & 3.96 & -0.36 \\
\hline V2 & 47.83 & 9.11 & 41.68 & 1.38 & 0.06 & 32.41 & 20.73 & 40.99 & 5.80 & 16.69 & 16.53 & -0.47 \\
\hline V3 & 50.06 & 42.64 & 4.90 & 2.41 & 1.02 & 18.27 & 1.24 & 77.45 & 2.02 & 3.19 & 3.09 & -1.61 \\
\hline V4 & 23.92 & 47.93 & 25.60 & 2.55 & 0.22 & 17.56 & 19.67 & 61.22 & 1.33 & 5.01 & 4.82 & -1.91 \\
\hline V5 & 22.19 & 66.71 & 9.67 & 1.42 & 0.53 & 19.88 & 2.68 & 74.92 & 2.00 & 1.80 & 1.70 & 0.10 \\
\hline V6 & 44.93 & 27.91 & 25.28 & 1.87 & 0.12 & 23.59 & 18.05 & 56.49 & 1.75 & 8.88 & 8.61 & -1.57 \\
\hline V7 & 40.22 & 40.30 & 16.05 & 3.44 & 0.29 & 10.31 & 9.28 & 75.77 & 4.36 & 2.98 & 2.74 & 0.24 \\
\hline V8 & 24.13 & 29.02 & 45.30 & 1.55 & 0.25 & 31.22 & 19.03 & 46.94 & 2.56 & 8.27 & 8.49 & 1.33 \\
\hline V9 & 47.42 & 17.28 & 33.51 & 1.80 & 0.11 & 36.51 & 23.39 & 37.46 & 2.53 & 9.26 & 9.58 & 1.69 \\
\hline $\mathrm{V} 10$ & 37.71 & 23.75 & 36.09 & 2.45 & 0.15 & 21.19 & 17.51 & 58.80 & 2.35 & 7.41 & 7.19 & -1.53 \\
\hline V11 & 18.32 & 47.19 & 31.14 & 3.35 & 0.17 & 6.65 & 36.09 & 56.41 & 0.68 & 12.21 & 12.72 & 2.07 \\
\hline V12 & 18.72 & 48.78 & 30.12 & 2.38 & 0.10 & 8.87 & 40.04 & 48.61 & 2.37 & 10.66 & 10.17 & -2.35 \\
\hline V13 & 33.95 & 47.63 & 16.33 & 2.09 & 0.18 & 16.97 & 16.15 & 65.31 & 1.40 & 5.88 & 5.98 & 0.88 \\
\hline V14 & 36.01 & 54.12 & 8.83 & 1.04 & 0.57 & 6.08 & 6.00 & 81.66 & 5.69 & 2.22 & 1.86 & 0.36 \\
\hline V15 & 64.93 & 21.69 & 10.61 & 2.77 & 0.70 & 22.38 & 13.64 & 57.98 & 5.31 & 1.84 & 1.51 & 0.33 \\
\hline V16 & 19.23 & 20.81 & 57.74 & 2.22 & 0.21 & 31.42 & 36.65 & 29.06 & 2.65 & 10.38 & 9.88 & -2.48 \\
\hline V17 & 24.35 & 16.26 & 56.79 & 2.60 & 0.17 & 30.83 & 40.84 & 26.15 & 2.00 & 8.85 & 9.15 & 1.64 \\
\hline V18 & 38.44 & 25.68 & 32.61 & 3.28 & 0.25 & 26.79 & 16.33 & 54.27 & 2.36 & 6.23 & 6.32 & 0.69 \\
\hline V19 & 42.39 & 17.12 & 37.76 & 2.73 & 0.35 & 21.16 & 12.55 & 62.50 & 3.44 & 3.20 & 3.32 & 1.87 \\
\hline V20 & 44.94 & 17.63 & 34.36 & 3.07 & 0.46 & 11.33 & 9.40 & 75.05 & 3.76 & 2.66 & 2.66 & -0.07 \\
\hline V21 & 39.61 & 23.91 & 34.49 & 1.98 & 0.51 & 13.19 & 13.30 & 70.44 & 2.55 & 5.23 & 5.32 & 0.86 \\
\hline V22 & 40.34 & 18.62 & 38.36 & 2.68 & 0.36 & 20.63 & 13.45 & 60.94 & 4.62 & 3.36 & 3.40 & 0.65 \\
\hline V23 & 39.42 & 22.75 & 35.30 & 2.54 & 0.28 & 20.53 & 14.61 & 58.31 & 6.27 & 3.44 & 3.42 & -0.26 \\
\hline V24 & 43.51 & 21.89 & 31.99 & 2.61 & 0.36 & 22.26 & 15.88 & 58.63 & 2.87 & 7.14 & 7.21 & 0.46 \\
\hline V25 & 50.32 & 17.95 & 28.51 & 3.21 & 0.52 & 8.72 & 7.84 & 78.58 & 4.35 & 3.48 & 3.45 & -0.48 \\
\hline V26 & 38.73 & 21.71 & 36.44 & 3.12 & 0.99 & 13.97 & 9.42 & 69.41 & 6.22 & 2.88 & 2.87 & -0.14 \\
\hline V27 & 38.39 & 28.24 & 29.54 & 3.83 & 0.38 & 8.01 & 6.65 & 76.41 & 8.55 & 2.49 & 2.50 & 0.27 \\
\hline V28 & 53.09 & 32.55 & 14.21 & 0.15 & 3.93 & 8.32 & 5.18 & 52.94 & 29.63 & 1.20 & 1.21 & 0.18 \\
\hline V29 & 61.08 & 21.79 & 16.43 & 0.70 & 0.51 & 7.00 & 5.81 & 66.75 & 19.93 & 1.44 & 1.43 & -0.06 \\
\hline V30 & 49.19 & 27.58 & 21.49 & 1.74 & 0.31 & 19.49 & 14.38 & 60.27 & 5.55 & 4.54 & 4.63 & 1.04 \\
\hline V31 & 46.43 & 14.80 & 36.45 & 2.31 & 0.31 & 27.39 & 20.93 & 44.70 & 6.67 & 6.86 & 6.96 & 0.67 \\
\hline V32 & 50.06 & 40.13 & 6.61 & 3.21 & 0.26 & 18.53 & 8.53 & 72.51 & 0.17 & 10.78 & 10.73 & -0.28 \\
\hline
\end{tabular}

Table 3 Hydrochemical faces of groundwater

\begin{tabular}{|c|c|c|c|}
\hline Faces & Sample ID & Number of samples & Percentage of samples \\
\hline $\mathrm{CaHCO}_{3}$ & $\begin{array}{c}\mathrm{V} 1, \mathrm{~V} 3, \mathrm{~V} 4, \mathrm{~V} 5, \mathrm{~V} 7, \mathrm{~V} 10, \mathrm{~V} 11, \mathrm{~V} 14, \mathrm{~V} 15, \mathrm{~V} 18, \mathrm{~V} 19, \mathrm{~V} 20, \mathrm{~V} 21, \mathrm{~V} 22, \\
\text { V24, V25, V26,V 27, V28, V29, V30, V32 }\end{array}$ & 22 & 68.75 \\
\hline $\mathrm{NaCl}$ & V16, V17 & 2 & 6.25 \\
\hline Mixed $\mathrm{CaNa} \mathrm{HCO}_{3}$ & Nill & Nill & Nill \\
\hline Mixed $\mathrm{CaMgCl}$ & V2, V6, V8, V9, V12, V13, V23, V31 & 8 & 25 \\
\hline $\mathrm{CaCl}$ & Nill & Nill & Nill \\
\hline $\mathrm{NaHCO}_{3}$ & Nill & Nill & Nill \\
\hline
\end{tabular}



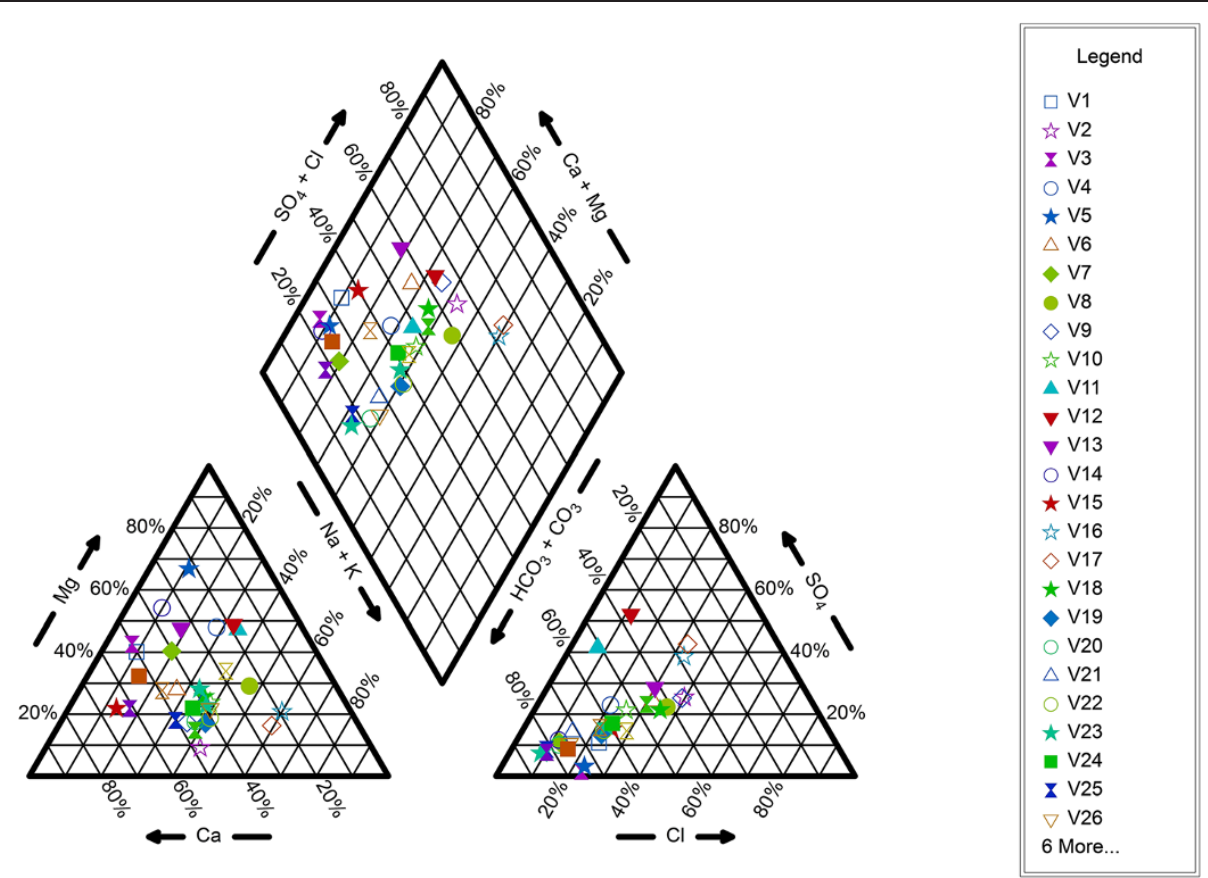

Figure 8 Piper diagram of the sampling points.

in groundwater. Exposure to high levels of arsenic can cause short-term or acute symptoms as well as longterm or chronic health effects. Arsenic in groundwater is found largely due to the minerals associated with previous volcanic activities dissolving from weathered rocks, ash and soils [22-24]. However, arsenic concentrations of greater than $10 \mu \mathrm{g} / \mathrm{L}$ (guideline value of World Health Organization for arsenic in drinking water [23]) were detected in the water supplies, especially in western district of the considered area (Figure 9). In Shadabad, Kondrud,

Table 4 Concentration of heavy metals in groundwater samples (in $\mu \mathrm{g} / \mathrm{L}$ )

\begin{tabular}{|c|c|c|c|c|c|c|c|c|c|c|c|}
\hline No & Villages & As & Al & B & $\mathrm{Ba}$ & $\mathrm{Be}$ & $\mathrm{Cr}$ & $\mathrm{Cu}$ & $\mathrm{Hg}$ & Sn & $\mathrm{Zn}$ \\
\hline $\mathrm{V} 1$ & Hervi & $<1$ & 1.89 & 450.16 & 56 & 4.85 & 23.28 & 29.00 & $<1$ & $<9$ & $<2$ \\
\hline V2 & Eskandar & $<1$ & 0.78 & 2074.76 & 105 & 4.83 & 22.26 & 31.72 & 6.50 & 19.57 & 2.87 \\
\hline V3 & Ligvan & $<1$ & 18.04 & 161.80 & 54 & 5.37 & 21.42 & 36.25 & $<1$ & $<9$ & $<2$ \\
\hline V4 & Shadabad & 22.43 & 15.81 & 500.82 & 34 & 5.61 & 20.35 & 37.68 & $<1$ & $<9$ & $<2$ \\
\hline V5 & Esparakhun & $<1$ & 147.11 & 61.84 & 8 & 5.54 & 21.48 & 42.30 & $<1$ & $<9$ & $<2$ \\
\hline V6 & Kondrud & 19.93 & 3.69 & 477.01 & 36 & 4.65 & 23.58 & 29.55 & $<1$ & $<9$ & $<2$ \\
\hline V7 & Dizaj leylikhani & $<1$ & 2.94 & 112.19 & 49 & 5.13 & 23.52 & 49.82 & $<1$ & $<9$ & 26.46 \\
\hline V8 & Asbes & 69.15 & 12.25 & 890.65 & 22 & 5.63 & 21.50 & 35.91 & $<1$ & $<9$ & 4.56 \\
\hline V9 & Asenjan & $<1$ & 4.31 & 504.23 & 99 & 4.84 & 23.42 & 30.55 & $<1$ & $<9$ & 131.10 \\
\hline V10 & Varanag & 24.19 & 4.13 & 519.78 & 52 & 5.10 & 23.21 & 28.83 & $<1$ & 10.80 & 25.26 \\
\hline V11 & Ola kandi & $<1$ & 2.36 & 1510.82 & 50 & 4.90 & 22.26 & 29.89 & $<1$ & 19.25 & 5.56 \\
\hline V12 & Esfahlan & 42.57 & 5.34 & 853.97 & 25 & 5.25 & 21.86 & 32.48 & $<1$ & 12.04 & 15.96 \\
\hline V13 & Anarjan & $<1$ & 7.76 & 277.97 & 69 & 4.86 & 23.46 & 29.47 & $<1$ & $<9$ & 10.54 \\
\hline V14 & Komanj olya & $<1$ & 101.39 & 45.01 & 14 & 5.33 & 22.49 & 32.91 & $<1$ & $<9$ & $<2$ \\
\hline V15 & Zinjanab & $<1$ & 19.74 & 51.45 & 5 & 5.29 & 22.26 & 36.72 & $<1$ & $<9$ & 5.59 \\
\hline V16 & Espiran1 & $<1$ & 14.26 & 708.16 & 40 & 4.76 & 23.84 & 29.62 & $<1$ & 9.38 & $<2$ \\
\hline V17 & Espiran2 & $<1$ & 2.27 & 1066.07 & 27 & 5.03 & 23.03 & 33.23 & $<1$ & 11.97 & 8.27 \\
\hline V18 & Khellejan & 36.07 & 3.04 & 734.88 & 47 & 5.19 & 22.86 & 33.70 & $<1$ & 10.57 & $<2$ \\
\hline- & Standard values & 10 & 100 & 2400 & 700 & 12 & 50 & 2000 & 6 & - & - \\
\hline
\end{tabular}




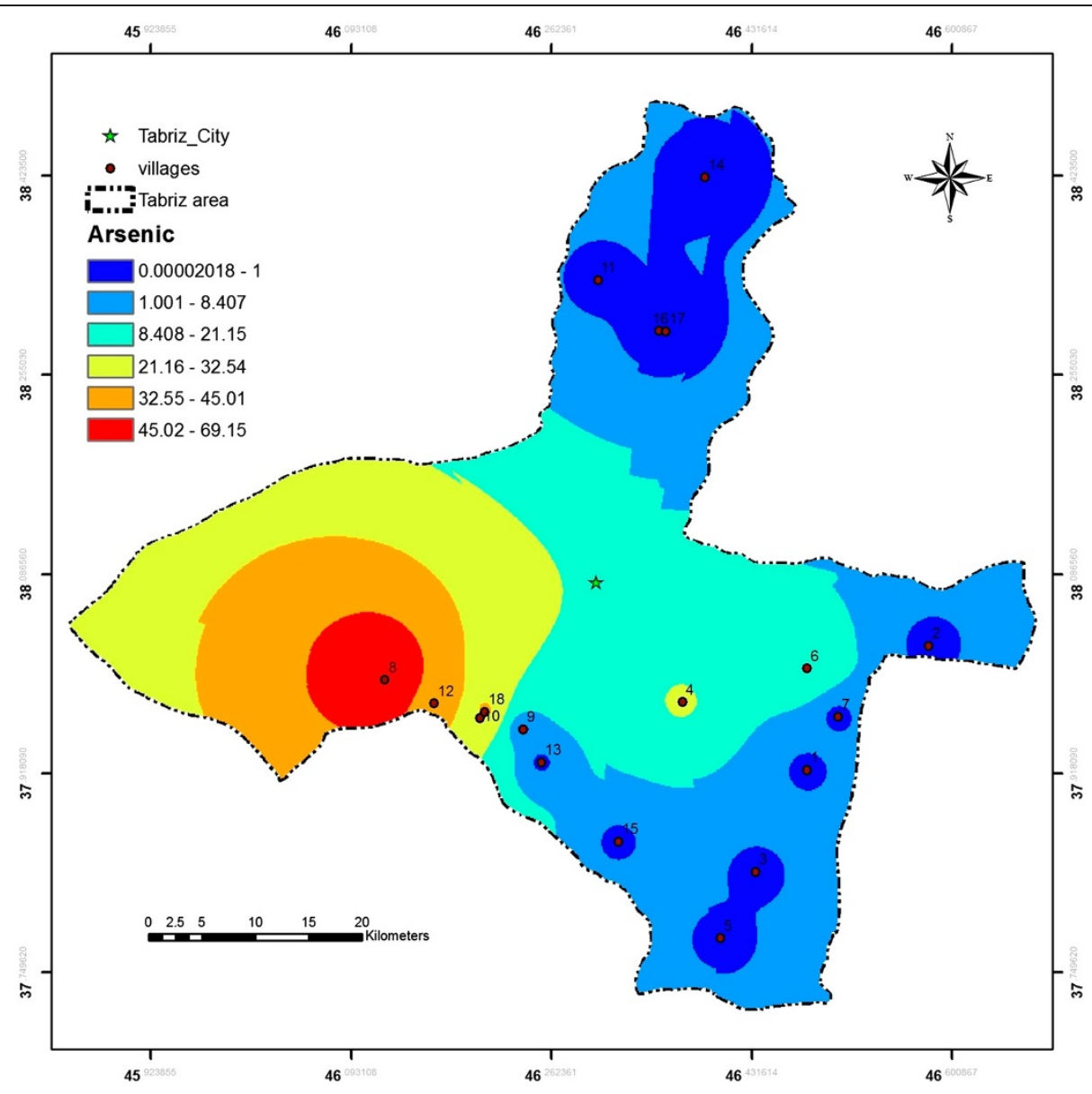

Figure 9 Arsenic distribution in the studied area.

Asbes, Varanag, Esfahlan and Khellejan villages, concentration of above $10 \mu \mathrm{g} / \mathrm{L}$ was found.

The presence of aluminum at concentrations exceeding $0.1-0.2 \mathrm{mg} / \mathrm{l}$ often leads to consumer complaints due to its deposition of aluminum hydroxide floc. There is no health-based guideline value for the concentration of aluminum in drinking water; however, aluminum concentrations of less than $0.1 \mathrm{mg} / \mathrm{L}$ are achievable in many circumstances [23]. Results of this investigation demonstrated that only 2 of the samples had aluminum concentrations of more than $0.1 \mathrm{mg} / \mathrm{L}$.

Inorganic mercury is a predominate form that is found in surface and groundwater, usually at concentrations of below $0.5 \mu \mathrm{g} / \mathrm{L}$; however, local mineral deposits may produce higher levels in groundwater [23]. As depicted in Table 4, only in one case, mercury concentration was above the guideline value $(6 \mu \mathrm{g} / \mathrm{L})$. Main problems caused by inorganic mercuric poisoning include liver and renal damage which lead to death [25]. Organicmethylmercury affects the central nervous system. Relatively few instances of elevated concentrations of mercury in groundwater have been reported, except when mercury is included among other contaminants in site-specific hazardous waste investigations or other studies of groundwater contamination in industrialized areas $[26,27]$.

Other heavy metal values presented in Table 4 were below the standard values.

\section{Statistical analysis}

Table 5 provides a matrix of correlation coefficients between quality parameters of the analyzed water samples along with heavy metals. Values of high correlation are specified in bold. As can be observed in the table, there was high correlation between total hardness, $\mathrm{Ca}^{2+}, \mathrm{HCO}_{3}{ }^{-}$, $\mathrm{Mg}^{2+} \mathrm{K}^{+}, \mathrm{Na}^{+}, \mathrm{SO}_{4}{ }^{2-}, \mathrm{Cl}^{-}, \mathrm{B}$ and $\mathrm{Sn}$. Furthermore, there was significant correlation between $\mathrm{Na}^{+}, \mathrm{SO}_{4}{ }^{2-}, \mathrm{NO}_{3}{ }^{2-}$ and B. The correlation between $\mathrm{Ca}^{2+}, \mathrm{NO}_{3}{ }^{2-}$ and $\mathrm{Hg}$ was also significant. As can be observed in Table 5 , the correlation between $\mathrm{Cr}$ and $\mathrm{Br}$ was negatively significant.

Table 6 demonstrates rotated factor loadings for water quality parameters. In KMO and Bartlett's Test, $\mathrm{p}<0.001$ with coefficient of 0.658 was significant. PCA showed that 3 components could cover $84.3 \%$ of the parameters. In 32 analyzed water samples, according to rotated component 
Table 5 Matrix of correlation coefficients between the analyzed parameters

\begin{tabular}{|c|c|c|c|c|c|c|c|c|c|c|c|c|c|c|c|c|c|c|c|c|c|c|}
\hline & EC & Hardness & $\mathrm{pH}$ & $\mathrm{Ca}^{2+}$ & $\mathrm{Mg}^{2+}$ & $\mathrm{Na}^{+}$ & $\mathrm{K}^{+}$ & $\mathrm{HCO}_{3}^{-}$ & $\mathrm{SO}_{4}^{2-}$ & $\mathrm{Cl}^{-}$ & $\mathrm{NO}_{3}{ }^{2-}$ & $\mathrm{F}^{-}$ & Al & As & $\mathrm{Hg}$ & B & $\mathrm{Ba}$ & $\mathrm{Br}$ & $\mathrm{Cr}$ & $\mathrm{Cu}$ & Sn & $\overline{Z n}$ \\
\hline EC & 1 & & & & & & & & & & & & & & & & & & & & & \\
\hline Hardness & 0.94 & 1 & & & & & & & & & & & & & & & & & & & & \\
\hline $\mathrm{pH}$ & -0.33 & -0.45 & 1 & & & & & & & & & & & & & & & & & & & \\
\hline $\mathrm{Ca}^{2+}$ & 0.82 & 0.81 & -0.25 & 1 & & & & & & & & & & & & & & & & & & \\
\hline $\mathrm{Mg}^{2+}$ & 0.65 & 0.76 & -0.49 & 0.24 & 1 & & & & & & & & & & & & & & & & & \\
\hline $\mathrm{Na}^{+}$ & 0.93 & 0.76 & -0.12 & 0.71 & 0.47 & 1 & & & & & & & & & & & & & & & & \\
\hline $\mathrm{K}^{+}$ & 0.82 & 0.78 & -0.281 & 0.51 & 0.73 & 0.73 & 1 & & & & & & & & & & & & & & & \\
\hline $\mathrm{HCO}_{3}^{-}$ & 0.87 & 0.95 & -0.36 & 0.72 & 0.78 & 0.67 & 0.78 & 1 & & & & & & & & & & & & & & \\
\hline $\mathrm{SO}_{4}^{2-}$ & 0.89 & 0.79 & -0.27 & 0.53 & 0.72 & 0.87 & 0.89 & 0.70 & 1 & & & & & & & & & & & & & \\
\hline $\mathrm{Cl}^{-}$ & 0.86 & 0.73 & -0.18 & 0.86 & 0.26 & 0.89 & 0.51 & 0.62 & 0.65 & 1 & & & & & & & & & & & & \\
\hline $\mathrm{NO}_{3}^{2-}$ & 0.53 & 0.41 & -0.01 & 0.71 & -0.10 & 0.55 & 0.20 & 0.29 & 0.35 & 0.61 & 1 & & & & & & & & & & & \\
\hline $\mathrm{F}^{-}$ & -0.01 & -0.04 & 0.15 & -0.07 & 0.01 & 0.05 & -0.05 & 0.03 & -0.02 & -0.01 & 0.07 & 1 & & & & & & & & & & \\
\hline Al & -0.49 & -0.51 & -0.01 & -0.44 & -0.28 & -0.40 & -0.57 & -0.50 & -0.41 & -0.38 & -0.24 & -0.18 & 1 & & & & & & & & & \\
\hline As & 0.17 & 0.15 & -0.29 & -0.04 & 0.29 & 0.14 & 0.11 & 0.23 & 0.09 & 0.08 & 0.01 & 0.11 & -0.20 & 1 & & & & & & & & \\
\hline $\mathrm{Hg}$ & 0.62 & 0.57 & -0.12 & 0.82 & -0.11 & 0.54 & 0.19 & 0.48 & 0.28 & 0.67 & 0.93 & -0.13 & -0.13 & -0.15 & 1 & & & & & & & \\
\hline B & 0.93 & 0.82 & -0.04 & 0.71 & 0.45 & 0.85 & 0.79 & 0.82 & 0.80 & 0.72 & 0.75 & 0.16 & -0.43 & 0.15 & 0.68 & 1 & & & & & & \\
\hline $\mathrm{Ba}$ & 0.53 & 0.61 & -0.17 & 0.76 & 0.03 & 0.37 & 0.34 & 0.54 & 0.24 & 0.60 & 0.56 & -0.01 & -0.50 & -0.26 & 0.55 & 0.45 & 1 & & & & & \\
\hline $\mathrm{Br}$ & -0.48 & -0.53 & 0.07 & -0.56 & -0.15 & -0.41 & -0.49 & -0.45 & -0.43 & -0.44 & -0.3 & 0.12 & 0.47 & 0.44 & -0.24 & -0.33 & -0.58 & 1 & & & & \\
\hline $\mathrm{Cr}$ & 0.13 & 0.01 & 0.00 & 0.23 & -0.13 & 0.18 & 0.18 & 0.05 & 0.12 & 0.23 & 0.04 & -0.22 & -0.32 & -0.32 & -0.08 & -0.05 & 0.33 & -0.81 & 1 & & & \\
\hline $\mathrm{Cu}$ & -0.48 & -0.53 & 0.33 & -0.42 & -0.34 & -0.41 & -0.47 & -0.50 & -0.45 & -0.38 & -0.12 & -0.13 & 0.33 & -0.02 & -0.01 & -0.38 & -0.32 & 0.57 & -0.33 & 1 & & \\
\hline Sn & 0.80 & 0.74 & -0.03 & 0.54 & 0.52 & 0.72 & 0.83 & 0.75 & 0.79 & 0.46 & 0.62 & 0.14 & -0.30 & -0.03 & 0.58 & 0.89 & 0.34 & -0.34 & 0.00 & -0.33 & 1 & \\
\hline Zn & 0.12 & 0.17 & 0.22 & 0.27 & -0.06 & 0.08 & 0.05 & 0.04 & 0.10 & 0.29 & 0.07 & -0.21 & -0.17 & -0.12 & -0.09 & -0.07 & 0.51 & -0.23 & 0.29 & -0.08 & -0.14 & 1 \\
\hline
\end{tabular}


Table 6 Rotated factor loadings of PCA application for water quality parameters

\begin{tabular}{cccc}
\hline Parameters & PC1 & PC2 & PC3 \\
\hline $\mathrm{Mg}^{2+}$ & $\mathbf{0 . 9 5 9}$ & -0.093 & -0.096 \\
$\mathrm{~K}^{+}$ & $\mathbf{0 . 8 5 3}$ & 0.289 & -0.008 \\
$\mathrm{HCO}_{\mathbf{3}}{ }^{-}$ & $\mathbf{0 . 8 2 0}$ & 0.411 & -0.074 \\
$\mathrm{SO}_{4}{ }^{2-}$ & $\mathbf{0 . 8 0 6}$ & 0.425 & 0.041 \\
Hardness & $\mathbf{0 . 7 9 6}$ & 0.526 & -0.166 \\
$\mathrm{EC}^{2-}$ & $\mathbf{0 . 7 3 4}$ & 0.673 & -0.040 \\
$\mathrm{NO}_{3}{ }^{2-}$ & -0.062 & $\mathbf{0 . 8 8 4}$ & 0.062 \\
$\mathrm{Ca}^{2+}$ & 0.330 & $\mathbf{0 . 8 6 7}$ & -0.168 \\
$\mathrm{Cl}^{-}$ & 0.371 & $\mathbf{0 . 8 5 6}$ & -0.017 \\
$\mathrm{Na}^{+}$ & 0.587 & $\mathbf{0 . 7 2 2}$ & 0.132 \\
$\mathbf{F}^{-}$ & 0.100 & -0.049 & $\mathbf{0 . 8 6 8}$ \\
$\mathbf{p H}$ & -0.477 & 0.049 & $\mathbf{0 . 5 7 5}$ \\
Eigenvalue & 7.244 & 1.780 & 1.092 \\
Variance \% & 60.366 & 14.833 & 9.102 \\
Cumulative \% & 60.366 & 75.199 & 84.301 \\
\hline
\end{tabular}

Numbers in bold indicate effective parameter in each PC.

matrix with 3 factor solution, PC1 accounted for more than $60.3 \%$ of total variance in the dataset and was loaded with magnesium, potassium, bicarbonate, sulfate, hardness and electric conductivity; i.e. these parameters demonstrated a similar behavior in groundwater. The second component (PC2) explaining $14.8 \%$ of total variance had strong positive loadings for nitrate, calcium, chloride and sodium. The third component (PC3) of PCA demonstrated that only $9.1 \%$ of total variation had positive loading of $\mathrm{pH}$ and fluoride.

In KMO and Bartlett's test for heavy metals, $\mathrm{p}<0.001$ with coefficient of 0.535 was considered significant. Principal component analysis showed that three components explained 72.39 percent of variance (Table 7). Using rotated component matrix with 3 factor solution, PC1 included boron, tin and mercury. PC2 contained copper, zinc, barium, chromium and beryllium and PC3 was loaded with arsenic and aluminum. In PC2, zinc, barium and chromium had reverse correlation with beryllium and copper and also there was reverse correlation between arsenic and aluminum in PC3.

Figure 10 demonstrated the dendrogram obtained by CA for water quality parameters. The figure indicates relationship and similarity between water resources. This dendrogram introduced four distinct groups as A, B, C and $\mathrm{D}$. Considering the location of different villages in the already presented quality maps, it can be observed that the villages grouped in cluster A $(3,5,7,14,15,19$, $20,22,23,25,26,27,28$ and 29) were mainly located in southern and northern parts of the studied area, the ones
Table 7 Rotated factor loadings of PCA application for heavy metals

\begin{tabular}{cccc}
\hline Parameters & PC1 & PC2 & PC3 \\
\hline B & $\mathbf{0 . 9 1 3}$ & -0.052 & -0.315 \\
Sn & $\mathbf{0 . 8 8 1}$ & -0.037 & -0.151 \\
$\mathbf{H g}$ & $\mathbf{0 . 8 7 1}$ & 0.007 & 0.214 \\
$\mathbf{B r}$ & -0.319 & $\mathbf{0 . 8 7 7}$ & -0.033 \\
$\mathbf{C r}$ & -0.103 & $\mathbf{- 0 . 8 5 9}$ & 0.047 \\
$\mathbf{B a}$ & 0.521 & $\mathbf{- 0 . 6 3 5}$ & 0.043 \\
Zn & -0.169 & $\mathbf{- 0 . 5 7 1}$ & 0.023 \\
$\mathbf{C u}$ & -0.278 & $\mathbf{0 . 5 0 4}$ & 0.411 \\
As & -0.099 & 0.406 & $-\mathbf{0 . 8 2 2}$ \\
Al & -0.274 & 0.495 & $\mathbf{0 . 6 1 3}$ \\
Eigenvalue & 3.790 & 2.165 & 1.283 \\
Variance \% & 37.905 & 21.650 & 12.835 \\
Cumulative \% & 37.905 & 59.555 & 72.390 \\
\hline
\end{tabular}

Numbers in bold indicate effective parameter in each PC.

grouped in cluster B $(1,4,10,13,18,21,24,30$ and 31) were distributed in western part of this area and those located in cluster $C(6,8,9,11,12,16,17$ and 32) were seen in different parts. Finally, the dendrogram clarified abnormality of the water sample from Eskandar village which constituted one group as cluster. As shown in Figure 10 and the results presented in tables and maps thus far, quality of water in this village was infelicitous compared to other villages, which could be due to the fact that water well of this village was located close to the river and in the agricultural area. Since water table level in this area had high intrusion of contaminants through river, which passed through the village and also agricultural drainage to this water table could be the probable reason for this difference.

Application of CA is useful for classifying groundwater in the whole region and makes adequately serving for spatial assessment possible in an optimal manner. Therefore, the number of sampling sites and cost in the monitoring network is reduced without losing any significance of the outcome $[28,29]$.

\section{Conclusion}

This study aimed to examine quality of drinking groundwater of rural communities in Tabriz. The results represented whether the water was suitable or unsuitable for drinking purposes in this area. It was also observed that villages like Eskandar and Olakandi had low quality drinking water. It is suggested to take some necessary measures for supplying desirable water to the people living in these villages.

The dominant cations were in the order of $\mathrm{Ca}^{2+}>\mathrm{Na}^{+}>$ $\mathrm{Mg}^{2+}>\mathrm{K}^{+}$and dominant anions are in the order of 


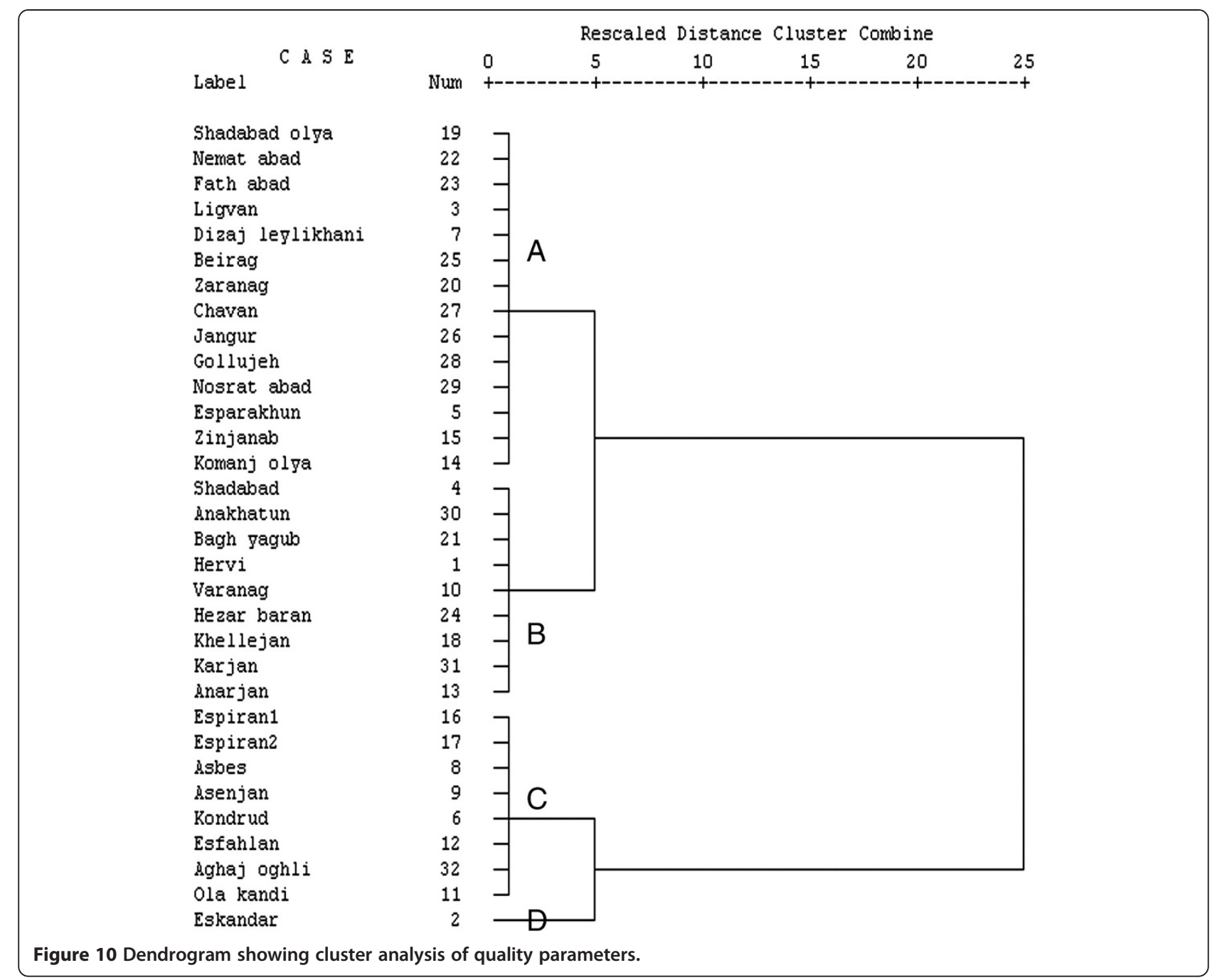

$\mathrm{HCO}_{3}{ }^{-}>\mathrm{Cl}^{-}>\mathrm{SO}_{4}{ }^{2-}>\mathrm{NO}_{3}{ }^{2-}$. So, hydrochemical faces of water were dominated by $\mathrm{Ca} \mathrm{HCO}_{3}$.

In this work, different multivariate statistical techniques were used to evaluate variations in groundwater quality. Cluster analysis grouped sampling sites to four clusters of similar water quality characteristics. Based on the obtained data, a future, optimal sampling strategy can be designed which could reduce the number of sampling sites and associated costs. Principle component analysis helped in identifying the factors or sources responsible for variations in water quality.

The results showed arsenic contamination in most of groundwater resources in the western areas. Universally, there are two sources for arsenic contamination in groundwater, which include geogenic source that is sometimes called background or natural and also anthropogenic sources [30,31]. The above-mentioned information can be useful for practical management of arsenic contamination problem and can provide an appropriate perspective for decision making and treatment strategies, especially for point of entry (POE) methods. Undoubtedly, application of different treatment methods that have been well discussed in the literature [32] should be considered an approach after pilot studies using real water samples for arsenic removal. Among the new methods for arsenic removal, ironamended biosand filters [33] and iron filter [34], electrochemical coagulation [35] and modified granular activated carbon [36,37] could be also considered. Therefore, it is recommended to conduct an extensive study in western areas of Tabriz to find source(s) of arsenic contamination, release mechanisms, distribution and periodic fluctuations of arsenic in the aquifers.

\section{Competing interests}

The authors declare that they have no competing interests.

\section{Authors' contributions}

The overall implementation of this study including design, experiments and data analysis were done by MM and MP and manuscript was drafted by MP. All authors have made extensive contribution into this study and critically reviewed the article. All authors read and approved the final manuscript. 


\section{Acknowledgment}

This article was a report resulted from the data set of M.Sc. thesis, registered in Tabriz University of Medical Sciences (TBZMED). The authors acknowledge East Azerbaijan Rural Water and Wastewater Company for funding the project and Student Research Committee of Tabriz University of Medical Sciences. We also appreciate Environmental Chemistry Laboratory of Faculty of Health, especially Mr. Mohammad Abedpour, Ms. Lida Kuhi and Ms. Parizad Faraji, for supportive contribution to the analyses of the water samples.

\section{Author details}

${ }^{1}$ Tabriz Health Services Management Research Center, Tabriz University of Medical Sciences, Tabriz, Iran. ${ }^{2}$ School of Health, Student research committee, Tabriz University of medical sciences, Tabriz, Iran. ${ }^{3}$ Department of Environmental Health Engineering, Tabriz University of Medical Sciences, Tabriz, Iran. ${ }^{4}$ Sahand University of Technology, Sahand, Iran. ${ }^{5}$ M.S. Graduated of Geographic Information Systems \& Remote Sensing, School of Geography, Tabriz University, Tabriz, Iran.

Received: 20 January 2014 Accepted: 17 June 2014

Published: 24 June 2014

\section{References}

1. Babiker IS, Mohamed MAA, Hiyama T, Kato K: A GIS-based DRASTIC model for assessing aquifer vulnerability in kakamigahara heights, gifu prefecture, central Japan. Sci Total Environ 2005, 345(1-3):127-140.

2. Mishra P, Behera P, Patel R: Contamination of water due to major industries and open refuse dumping in the steel city of Orissa-a case study. J Environ Sci Eng 2005, 47(2):141-154.

3. Moel PJD, Verberk JQJC, Dijk JCV: Drinking Water Principles and Practices. Singapore: World Scientific Publishing Company; 2007.

4. Arumugam $\mathrm{K}$, Elangovan $\mathrm{K}$ : Hydrochemical characteristics and groundwater quality assessment in Tirupur Region, Coimbatore District, Tamil Nadu, India. Environ Geol 2009, 58:1509-1520.

5. Edmunds WM, Shand P, Hart P, Ward RS: The natural (baseline) quality of groundwater: a UK pilot study. Sci Total Environ 2003, 310(1-3):25-35.

6. Nickson RT, McArthur JM, Shrestha B, Kyaw-Myint TO, Lowry D: Arsenic and other drinking water quality issues, Muzaffargarh District, Pakistan. Appl Geochem 2005, 20(1):55-68.

7. Baghvand A, Nasrabadi T, Bidhendi GN, Vosoogh A, Karbassi A, Mehrdadi N: Groundwater quality degradation of an aquifer in Iran central desert. Desalination 2010, 260:264-275.

8. Shamsi UM: GIS Applications for Water, Wastewater, and Stormwater Systems. United States of America: Taylor \& Francis; 2005.

9. Assaf H, Saadeh M: Assessing water quality management options in the Upper Litani Basin, Lebanon, using an integrated GIS-based decision support system. Environ Model Softw 2008, 23(10-11):1327-1337.

10. An Y, Wang Y, Zhang $H$, Wu X: GIS-based suitability assessment for shallow groundwater development in zhangye Basin. Procedia Environ Sci 2012, 12(0):1397-1403. Part B.

11. Balakrishnan P, Saleem A, Mallikarjun ND: Groundwater quality mapping using geographic information system (GIS): a case study of Gulbarga City, Karnataka, India. Afr J Environ Sci Technol 2011, 5(12):1069-1084.

12. Jamshidzadeh Z, Mirbagheri SA: Evaluation of groundwater quantity and quality in the Kashan Basin, Central Iran. Desalination 2011, 270:23-30.

13. Hoang TH, Bang S, Kim K-W, Nguyen MH, Dang DM: Arsenic in groundwater and sediment in the Mekong River delta, Vietnam. Environ Pollut 2010, 158(8):2648-2658.

14. Mende A, Astorga A, Neumann D: Strategy for groundwater management in developing countries: a case study in northern Costa Rica. J Hydrol 2007, 334(1-2):109-124.

15. APHA-AWWA-WPCF: Standard Methods for Examination of Water and Wastewater. 21st edition. United States of America;: American Water Work Association; 2005.

16. Shekhar S, Xiong H: Encyclopedia of GIS. Unites States of America: Springer; 2008.

17. Kazi TG, Arain MB, Jamali MK, Jalbani N, Afridi HI, Sarfraz RA, Baig JA, Shah AQ: Assessment of water quality of polluted lake using multivariate statistical techniques: a case study. Ecotoxicol Environ Saf 2009, 72(2):301-309.

18. Li S, Li J, Zhang Q: Water quality assessment in the rivers along the water conveyance system of the Middle Route of the South to North Water
Transfer Project (China) using multivariate statistical techniques and receptor modeling. J Hazard Mater 2011, 195:306-317.

19. Vieira JS, Pires JCM, Martins FG, Vilar VJP, Boaventura RAR, Botelho CMS: Surface water quality assessment of Lis river using multivariate statistical methods. Water Air Soil Pollutant 2012, 223(9):5549-5561.

20. Zhao Y, Xia XH, Yang ZF, Wang F: Assessment of water quality in Baiyangdian lake using multivariate statistical techniques. Procedia Environ Sci 2012, 13:1213-1226.

21. Hussain M, Ahmed SM, Abderrahman W: Cluster analysis and quality assessment of logged water at an irrigation project, eastern Saudi Arabia. J Environ Manag 2008, 86(1):297-307.

22. Mosaferi M, Yunesian M, Dastgiri S, Mesdaghinia A, Esmailnasab N: Prevalence of skin lesions and exposure to arsenic in drinking water in Iran. Sci Total Environ 2008, 390(1):69-76.

23. WHO: Guidelines for Drinking-water Quality. Fourth edition. Geneva, Switzerland: World Health Organization; 2011.

24. Christodoulidou M, Charalambous C, Aletrari M, Nicolaidou Kanari P, Petronda A, Ward Nl: Arsenic concentrations in groundwaters of Cyprus. J Hydrol 2012, 468-469:94-100.

25. Gray NF: Drinking Water Quality Problems and Solutions. 2nd edition. New York: Cambridge University Press; 2008.

26. Julia L, Barringer J, MacLeod CL: Relation of Mercury to Other Chemical Constituents in Groundwater in the Kirkwoodcohansey Aquifer System, New Jersey Coastal Plain, and Mechanisms for Mobilization of Mercury from Sediments to Groundwater. West Trenton, New Jersey: U.S. Geological Survey; 2001.

27. Barringer JL, Szabo Z, Schneider D, Atkinson WD, Gallagher RA: Mercury in ground water, septage, leach-field effluent, and soils in residential areas, New Jersey coastal plain. Sci Total Environ 2006, 361(1-3):144-162.

28. Kim J-H, Yum B-W, Kim R-H, Koh D-C, Cheong T-J, Lee J, Chang H-W: Application of cluster analysis for the hydrogeochemical factors of saline groundwater in Kimje, Korea. Geosci J 2003, 7(4):313-322.

29. Kim J-H, Kim R-H, Lee J, Cheong T-J, Yum B-W, Chang H-W: Multivariate statistical analysis to identify the major factors governing groundwater quality in the coastal area of Kimje, South Korea. Hydrol Process 2004, 19(6):1261-1276.

30. Smedley PL, Kinniburgh DG: A review of the source, behaviour and distribution of arsenic in natural waters. Appl Geochem 2002, 17(5):517-568,

31. Bissen M, Frimmel FH: Arsenic - a review. Part I: occurrence, toxicity, speciation, mobility. Acta Hydrochim Hydrobiol 2003, 31(1):9-18.

32. Bissen M, Frimmel FH: Arsenic - a review. Part II: oxidation of arsenic and its removal in water treatment. Acta Hydrochim Hydrobiol 2003, 31(2):97-107.

33. Noubactep C, Temgoua E, Rahman MA: Designing iron-amended biosand filters for decentralized safe drinking water provision. CLEAN - Soil, Air, Water 2012, 40(8):798-807.

34. Noubactep C, Schöner A, Woafo P: Metallic iron filters for universal access to safe drinking water. CLEAN - Soil, Air, Water 2009, 37(12):930-937.

35. Vasudevan S, Lakshmi J, Sozhan G: Studies on the removal of arsenate by electrochemical coagulation using aluminum alloy anode. CLEAN - Soil, Air, Water 2010, 38(5-6):506-515.

36. Mondal P, Mohanty B, Balomajumder C: Treatment of arsenic contaminated groundwater using calcium impregnated granular activated carbon in a batch reactor: optimization of process parameters. CLEAN - Soil, Air, Water 2010, 38(2):129-139.

37. Mondal $P$, Mohanty B, Majumder CB: Removal of arsenic from simulated groundwater using GAC-Ca in batch reactor: kinetics and equilibrium studies. CLEAN - Soil, Air, Water 2012, 40(5):506-514.

doi:10.1186/2052-336X-12-99

Cite this article as: Mosaferi et al:: Quality modeling of drinking groundwater using GIS in rural communities, northwest of Iran. Journal of Environmental Health Science \& Engineering 2014 12:99. 